However, this is the first to ascertain normal JRAs for Dachshunds, a small and chondrodystrophic breed, which interestingly are comparable to other larger, nonchondrodystrophic breeds. Angular limb deformities are challenging cases to manage, an established reference range can help optimise surgical outcome, particularly when the contralateral limb is abnormal. To the authors knowledge, the reliability of this method to establish tibia JRAs has not been previously determined. The good ICC supports this method of calculating tibial JRAs.

\section{Comparison of accuracy of free- hand, fluoroscopically guided, and aiming device assisted drilling}

\section{Ian Faux, Jon Hall, Dylan Clements, John Ryan}

Hospital for Small Animals, University of Edinburgh, Edinburgh, United Kingdom

\section{OBJECTIVES}

To evaluate the accuracy of drilling using augmented drilling methods (fluoroscopy, aiming device) compared to free-hand drilling

- To evaluate the accuracy of drilling when performed by surgeons of different experience levels and when drilling is performed at an angle
To assess the impact which drilling method and surgeon experience has on the duration of the drilling procedure.

\section{METHODS}

Three ECVS diplomates and three qualified veterinary surgeons without primary orthopaedic experience drilled into synthetic bone using three different methods (freehand, fluoroscopy, aiming device) at three different angles $\left(0^{\circ}, 10^{\circ}\right.$ and $\left.20^{\circ}\right)$ aiming for a target (Point C). The distance between the drill exit hole and Point $C$ and the duration of drilling was recorded.

\section{RESULTS}

The two augmented methods were more accurate than free-hand drilling, with the aiming device being the most accurate. Increased angulation of drilling decreased the drilling accuracy. Surgeon experience did not impact on drilling accuracy, but it enabled faster completion of the drilling procedure.

\section{STATEMENT (CONCLUSIONS)}

The use of augmented drilling methods improved the accuracy of drilling and surgeons should consider their use when drilling anatomical regions where the margin of error is small.

\section{Medial epicondylar fissure fracture as a complication during stabilisation of humeral intracondylar fissures}

\section{Georgia Jenkins, Andy Moores}

Anderson Moores Veterinary Specialists, Winchester, United Kingdom

\section{OBJECTIVES}

To report the incidence of medial epicondylar fissure fracture occurring during transcondylar screw placement for stabilisation of humeral intracondylar fissure, and to determine if this increased the risk of subsequent complications.

\section{METHODS}

Case records were searched to identify dogs that had undergone surgery for humeral intracondylar fissure. Cases were included if both pre-and post-operative imaging were available, and if re-examination was performed a minimum of six weeks post-operatively. Post-operative imaging was evaluated for presence of medial epicondylar fissure fractures, information from case records and radiographs were used to identify post-operative complications. Statistical analysis was performed using Fisher's test, and Odds Ratio.

\section{RESULTS}

Eighty-four elbows underwent transcondylar screw placement; medial epicondylar fissure fracture occurred in nine elbows at the time of surgery (10.7\%). The most common transcondylar screw type was a $4.5 \mathrm{~mm}$ shaft screw $(71 / 84,84.5 \%)$. All cases which developed medial epicondylar fissure fracture did so after placement of a $4.5 \mathrm{~mm}$ shaft screw; this was not a significant association (Fisher's test $(p=0.3425)$ ). Of the 77 dogs which returned for re-examination, eight had had medial epicondylar fissure fracture. Complications were identified in 16 cases; 


\section{Oral presentations}

screw loosening was the most frequent (nine cases) and was the only complication seen in dogs with fissure fracture (three cases). Presence of medial epicondylar fissure fracture and development of screw loosening were significantly associated (Fisher's test ( $p=0.0468$ ), Odds Ratio $6.3(p=0.0296))$.

\section{STATEMENT (CONCLUSIONS)}

Medial epicondylar fissure fracture is a previously unreported complication following placement of transcondylar screws. Their occurrence was significantly associated with subsequent screw loosening.

\section{Computed}

Tomographic (CT)

Measurement of

Femoral Trochlear

Groove Depth in Brachycephalic Dogs

\section{Allie Matchwick ${ }^{1}$, Janis Bridges ${ }^{2}$, Ben Mielke ${ }^{3}$, Matthew Pead ${ }^{3}$, Andrew Phillips ${ }^{3}$, Richard Meeson ${ }^{3}$}

1 Anderson Moores Veterinary Specialists, Winchester, United Kingdom

2 Massey University, Palmerston North, New Zealand

3 Royal Veterinary College, London, United Kingdom

\section{OBJECTIVES}

To determine whether there is a difference in the trochlear groove depth of brachycephalic and non-brachycephalic dogs, and to compare between different brachycephalic breeds with varying risk for medial patellar luxation.

\section{METHODS}

Retrospective blinded study using a previously validated ratio (T/P) of maximal trochlear groove depth $(T)$ and maximal patella craniocaudal thickness $(P)$ measured on CT, to assess trochlear groove depth in Pugs, French Bulldogs and English Bulldogs without clinical patellar luxation. Combined brachycephalic T/P was compared to published data on mongrel non-brachycephalic dogs. The effect of breed on T/P was assessed using one-way linear regression models.

\section{RESULTS}

The mean T/P is affected by breed $(p<0.001)$. There is a significant difference between the T/P of Pugs (0.45) and French Bulldogs (0.38) $(p<0.001)$ and between Pugs and English Bulldogs (0.4) $(P<0.001)$. Mean T/P is significantly reduced in the brachycephalic dog breeds $(p<0.001)$.

\section{STATEMENT (CONCLUSIONS)}

The trochlea groove varies by breed, and was more shallow in the brachycephalic dogs at increased risk of patellar luxation. A shallow groove may be a breed driven characteristic predisposing to patellar luxation. Trochlear recession to achieve a patellar coverage of $50 \%$ (T/P 0.5) has been previously recommended for patellar luxation correction but may be excessive considering breed normal depth.

\section{Apprentices becoming} masters: learning curves and subsequent long-term outcomes for two surgeons performing lateral fabellar suture for cranial cruciate ligament rupture

\section{David Mills, Millie Wilder}

PDSA Thamesmead Pet Animal Hospital, London, United Kingdom

\section{OBJECTIVES}

- Define the number of supervised operations required for two surgeons to become independently competent in lateral fabellar suture (LFS) placement for cranial cruciate ligament rupture (CCLR)

- Detail long-term outcomes for both surgeons following achievement of independent competence, and compare to their surgeonteacher's outcomes.

\section{METHODS}

Retrospective study detailing cases of CCLR treated surgically by LFS by two surgeons (S1, 1 year graduated; S2, 8 years graduated) under the supervision of a 